\title{
Communicating Remote Gaussian Sources over Gaussian Multiple Access Channels
}

\author{
Amos Lapidoth \\ Signal and Information Processing Laboratory \\ ETH Zürich, \\ CH-8092 Zürich, Switzerland \\ amos.lapidoth@isi.ee.ethz.ch
}

\author{
I-Hsiang Wang \\ Wireless Foundations \\ University of California at Berkeley, \\ Berkeley, California 94720, USA \\ insiang@eecs.berkeley.edu
}

\begin{abstract}
We study a multiple-terminal joint source-channel coding problem, where two remote correlated Gaussian sources are transmitted over a Gaussian multiple-access channel with two transmitters. Each transmitter observes one of the sources contaminated in Gaussian noise. The receiver wishes to reconstruct both sources. We derive necessary conditions and sufficient conditions for the receiver to be able to reconstruct the sources with given expected squared-error distortions. These conditions establish the optimality of uncoded transmission below some signal-to-noise ratio (SNR) threshold, and they also establish the high-SNR asymptotics. To achieve the latter, a coding scheme is proposed that superimposes analog uncoded transmission and digital combined source-channel Gaussian vector quantization.
\end{abstract}

\section{INTRODUCTION}

Shannon's source-channel separation theorem [1] states that the problem of communicating a source over a noisy channel can be decomposed into lossy source compression and noisy channel coding without any penalty in performance. The classical result leads to an elegant paradigm of designing communication systems, namely, source-channel separation. Such separation, however, no longer holds in networks and multi-user settings, and the optimal code design involves jointly considering source and channel coding. In the state of the art, only a limited list of joint source-channel coding problems in the multi-user setting are studied and solved. One of the solved problems is sending jointly Gaussian sources over a Gaussian broadcast channel [2][3], where the achievable distortion region is characterized completely. On the other hand, the problem of sending jointly Gaussian sources over a Gaussian multiple access channel is still open. In [4] it was shown that uncoded transmission is optimal below some signal-to-noise threshold. A necessary condition and several sufficient conditions are also derived, and the high SNR asymptotic is characterized for the optimal achievable distortion. To date, the exact distortion region is unknown.

In some applications the sources are corrupted by noise before being observed by the encoders, and the reconstructions are corrupted after being produced by the decoders. Such a problem was first investigated by Dobrushin and Tsybakov [5] who showed that the problem can be reduced to the classical one considered by Shannon [1] with a modified distortion. Wolf and Ziv [6] investigated the same setting with squarederror distortion. They showed that the problem reduces to the classical one with the same distortion measure but with the reconstruction target now being the conditional expectation of the remote source given the observation. Moreover, the optimal scheme is the same as the scheme in a system where the observation is the conditional expectation of the remote source given the observation. This result implies the optimality of estimation-coding separation, where the estimation of the remote source is separated from the joint source-channel coding.

A natural question is whether estimation-coding separation is also optimal in multi-user scenarios (with squared-error distortion). In this paper we study the problem of sending remote Gaussian sources over a Gaussian MAC and answer this question in the negative. This conclusion is not surprising because the observations are correlated and hence the estimation at the encoder based on its own observation is not sufficient. It turns out that-unlike the direct source-channel coding problem studied in [4], where a vector quantization scheme suffices to achieve the high SNR asymptotic distortion—such a scheme does not suffice in the remote setting. Instead, we propose a superposition of vector quantization and uncoded scheme.

The rest of the paper is organized as follows. We first formulate our problem in Section II, briefly review Wolf and Ziv's result for the point-to-point case, and explain why the proof cannot be extended to our scenario. Then we show that our problem is equivalent to one where the reconstruction targets are the conditional expectation of the remote sources given both observations. Since our setting is Gaussian, these conditional expectations are linear combinations of the observations. In Section III we derive a necessary and two sufficient conditions for the achievability of a distortion pair, and we show that uncoded scheme is optimal below some SNR threshold. We also show that a superposition scheme is asymptotically optimal at high channel SNR. Some concluding remarks are presented in Section IV.

\section{Preliminaries AND Problem Formulation}

\section{A. Problem Formulation}

We consider the set-up depicted in Fig. 1(a), where the remote sources are jointly Gaussian with zero mean and covariance matrix $K_{w}=\left[\begin{array}{cc}\sigma_{1}^{2} & \rho \sigma_{1} \sigma_{2} \\ \rho \sigma_{1} \sigma_{2} & \sigma_{2}^{2}\end{array}\right]$, and Encoder $i$ 's 
observation $s_{i}$ is

$$
s_{i}=w_{i}+z_{i}, z_{i} \sim \mathcal{N}\left(0, N_{i}\right), i=1,2,
$$

where the noises $z_{1}, z_{2}$ are independent. Without loss of generality, we assume that $\rho \geq 0$ as in [4].

The encoders communicate with the decoder via a Gaussian multiple access channel, with power constraint $P_{i}$, for $i=1,2$, and the additive Gaussian noise $z \sim \mathcal{N}\left(0, N_{o}\right)$. We focus on the matched-bandwidth set-up, where the sources produce symbols at the same rate at which the channel is used. Throughout this paper, we use boldface $\mathbf{x}$ to denote a sequence of i.i.d. realizations of $x$ 's, with an implicit block length which can be arbitrarily large.

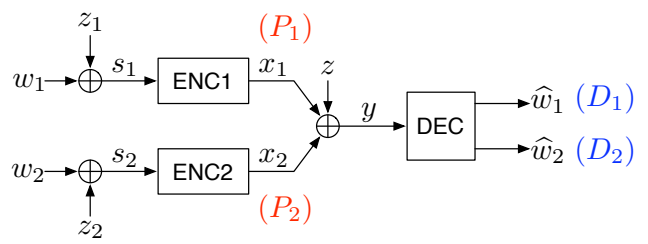

(a) Remote Source Setting

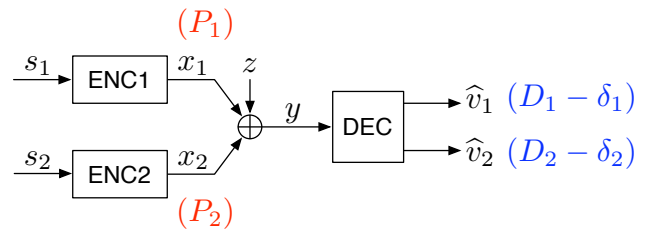

(b) Equivalent Setting

Fig. 1. System Models

\section{B. Point-to-point case: Decomposition}

Before dealing with our problem, we first revisit the pointto-point remote joint source-channel coding problem considered in [5] and [6].

Consider the problem where a remote source $(w)$ is observed by an encoder (as $s$ ) which communicates to a decoder through a point-to-point memoryless channel (input $x$ and output $y$ ) so that the decoder can reconstruct the source $(\widehat{w})$ up to a certain distortion. If the distortion measure is squared-error distortion, we can decompose the end-to-end distortion as [6]

$$
\begin{aligned}
\mathrm{E}\left[\|\mathbf{w}-\widehat{\mathbf{w}}\|^{2}\right] & =\mathrm{E}\left[\|\mathbf{w}-\mathbf{v}\|^{2}\right]+\mathrm{E}\left[\|\mathbf{v}-\widehat{\mathbf{w}}\|^{2}\right] \\
& =\operatorname{MMSE}[\mathbf{w} \mid \mathbf{s}]+\mathrm{E}\left[\|\mathbf{v}-\widehat{\mathbf{w}}\|^{2}\right]
\end{aligned}
$$

where $\mathbf{v}=\mathrm{E}[\mathbf{w} \mid \mathbf{s}]$. This follows because $\mathbf{w} \leftrightarrow \mathbf{v} \leftrightarrow \widehat{\mathbf{w}}$ forms a Markov chain for all encoding and decoding functions. From the above decomposition, we see that the optimal encoding is to first estimate the remote source $\mathbf{w}$ from the observation $\mathbf{s}$, and to then design an optimal source-channel code for the estimator output $\mathbf{v}$.

Why need the above decomposition not hold in our multiterminal setting? The key to the decomposition is the Markov chain $\mathbf{w} \leftrightarrow \mathrm{E}[\mathbf{w} \mid \mathbf{s}] \leftrightarrow \widehat{\mathbf{w}}$. In our setting, the corresponding Markov chain is $\mathbf{w}_{i} \leftrightarrow \mathrm{E}\left[\mathbf{w}_{i} \mid \mathbf{s}_{1}, \mathbf{s}_{2}\right] \leftrightarrow \widehat{\mathbf{w}}_{i}$, for $i=1,2$. Encoder $i$, however, only has access to $\mathbf{s}_{i}$, and hence cannot construct $\mathrm{E}\left[\mathbf{w}_{i} \mid \mathbf{s}_{1}, \mathbf{s}_{2}\right]$. If we replace $\mathrm{E}\left[\mathbf{w}_{i} \mid \mathbf{s}_{1}, \mathbf{s}_{2}\right]$ by
$\mathrm{E}\left[\mathbf{w}_{i} \mid \mathbf{s}_{i}\right]$, then the Markov condition need no longer hold, and hence nor the decomposition.

Under the Gaussian framework considered in this work, since the optimal estimator is linear, the MMSE estimation $\mathrm{E}\left[\mathbf{w}_{i} \mid \mathbf{s}_{i}\right]$ is just a scaled version of the observation $\mathbf{s}_{i}$. Therefore, to understand whether the decomposition holds or not, it suffices to study whether we can directly reuse the encoding/decoding blocks in [4] which are shown to be optimal in various regimes for the direct joint source channel coding problem. It turns out that the answer is no.

For simplicity, in the rest of this paper we shall focus on the symmetric set-up, where $\sigma_{1}^{2}=\sigma_{2}^{2}=: \sigma^{2}, N_{1}=N_{2}=: N$, and $P_{1}=P_{2}=: P$. In this seetting $\eta_{\mathrm{c}}:=P / N_{o}>0$ and $\eta_{\mathrm{s}}:=\sigma^{2} / N>0$ denote the signal-to-noise ratio in the channel and in observing the source respectively.

\section{Equivalence}

To facilitate the discussion, we begin by showing that the remote Gaussian joint source-channel coding problem is equivalent to a direct one with linear functions as reconstruction target.

Lemma 1 (Equivalence): The above problem is equivalent to reconstructing $v_{1}$ and $v_{2}$ at the receiver with distortion $D_{1}-$ $\delta_{1}$ and $D_{2}-\delta_{2}$ respectively, where

$$
v_{i}=\mathrm{E}\left[w_{i} \mid s_{1}, s_{2}\right], \delta_{i}=\operatorname{MMSE}\left[w_{i} \mid s_{1}, s_{2}\right], i=1,2 .
$$

Proof: The proof uses the same technique as in Section VI-B of [7] for the remote source coding problem, and is pretty straightforward: due to the Markov chain $\mathbf{w}_{i} \leftrightarrow$ $\mathrm{E}\left[\mathbf{w}_{i} \mid \mathbf{s}_{1}, \mathbf{s}_{2}\right] \leftrightarrow \widehat{\mathbf{w}}_{i}$, the distortion can be decomposed as

$$
\begin{aligned}
N D_{i} & =\mathrm{E}\left[\left\|\mathbf{w}_{i}-\widehat{\mathbf{w}}_{i}\right\|^{2}\right]=\mathrm{E}\left[\left\|\mathbf{w}_{i}-\mathbf{v}_{i}\right\|^{2}\right]+\mathrm{E}\left[\left\|\mathbf{v}_{i}-\widehat{\mathbf{w}}_{i}\right\|^{2}\right] \\
& =\operatorname{MMSE}\left[\mathbf{w}_{i} \mid \mathbf{s}_{1}, \mathbf{s}_{2}\right]+\mathrm{E}\left[\left\|\mathbf{v}_{i}-\widehat{\mathbf{w}}_{i}\right\|^{2}\right] \\
& =N \delta_{i}+\mathrm{E}\left[\left\|\mathbf{v}_{i}-\widehat{\mathbf{w}}_{i}\right\|^{2}\right], i=1,2,
\end{aligned}
$$

and hence the equivalence.

For the symmetric Gaussian setting treated in this paper, we note that $v_{1}=\alpha s_{1}+\beta s_{2}, v_{2}=\beta s_{1}+\alpha s_{2}$, where

$$
\alpha=\frac{\eta_{\mathrm{s}}\left(1+\left(1-\rho^{2}\right) \eta_{\mathrm{s}}\right)}{1+2 \eta_{\mathrm{s}}+\left(1-\rho^{2}\right) \eta_{\mathrm{s}}^{2}}, \beta=\frac{\rho \eta_{\mathrm{s}}}{1+2 \eta_{\mathrm{s}}+\left(1-\rho^{2}\right) \eta_{\mathrm{s}}^{2}} .
$$

Note that $\alpha=\beta \Longleftrightarrow 1+\left(1-\rho^{2}\right) \eta_{\mathrm{s}}=\rho \Longleftrightarrow(1-$ $\rho)\left(1+(1+\rho) \eta_{\mathrm{s}}\right)=0 \Longleftrightarrow \rho=1$, since $\eta_{\mathrm{s}}>0$. For the case of $\rho=1$, [8] provides the complete solution and shows that uncoded transmission is optimal. Therefore, we shall consider the case $\alpha \neq \beta$.

We also note that scaling $\alpha$ and $\beta$ by a constant will simply scale the distortion by the square of the constant, and will not change the optimal design of the coding scheme. Therefore for mathematical convenience, in the rest of this paper we shall without loss of generality consider the scaled version of $\alpha$ and $\beta$ such that the variance of $v_{1}$ and $v_{2}$ is equal to the original source: ( $\rho_{s}$ is the correlation coefficient of $\left(s_{1}, s_{2}\right)$ )

$$
\begin{aligned}
& \sigma_{s}^{2}=\operatorname{Var}\left[v_{i}\right]=\left(\alpha^{2}+\beta^{2}+2 \rho_{s} \alpha \beta\right) \sigma_{s}^{2} \\
& \Longleftrightarrow \alpha^{2}+\beta^{2}+2 \rho_{s} \alpha \beta=1 .
\end{aligned}
$$




\section{MAin Results}

By Lemma 1, we now consider the setting with two correlated Gaussian sources $s_{1}$ and $s_{2}$ with covariance matrix $K_{s}=\sigma_{s}^{2}\left[\begin{array}{cc}1 & \rho_{s} \\ \rho_{s} & 1\end{array}\right]$, each of which is observed by an encoder individually. The two encoders communicate to a decoder through a symmetric Gaussian MAC with power constraints $P$ at both transmitters and additive noise variance $N_{o}$. The decoder aims to reconstruct $v_{1}=\alpha s_{1}+\beta s_{2}$ and $v_{2}=$ $\beta s_{1}+\alpha s_{2}$ up to distortion $D^{s}$, where $\alpha>0, \beta>0, \alpha \neq \beta$, and they satisfy (1). See Fig. 2(a) for an illustration.

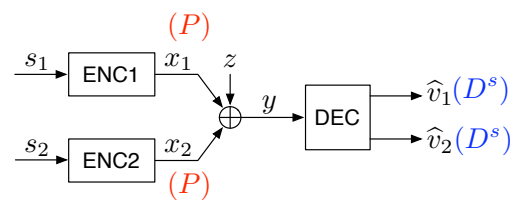

(a) System Model

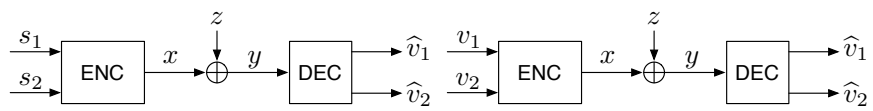

(b) Enhanced System

(c) Equivalent Enhanced System

Fig. 2. Symmetric Setting Considered

Before proceeding, we first compute a couple of parameters specified by the new problem of reconstructing linear functions $v_{1}$ and $v_{2}$, as follows:

$$
\begin{aligned}
\sigma_{v}^{2} & :=\operatorname{Var}\left[v_{1}\right]=\operatorname{Var}\left[v_{2}\right] \stackrel{(\mathrm{a})}{=} \sigma_{s}^{2} \\
\rho_{v} & :=\frac{\operatorname{Cov}\left[v_{1}, v_{2}\right]}{\sqrt{\operatorname{Var}\left[v_{1}\right] \operatorname{Var}\left[v_{2}\right]}}=2 \alpha \beta+\left(\alpha^{2}+\beta^{2}\right) \rho_{s} \\
& \stackrel{(\mathrm{b})}{=} \rho_{s}+2 \alpha \beta\left(1-\rho_{s}^{2}\right),
\end{aligned}
$$

where (a) and (b) are both due to condition (1).

\section{A. Outer Bound}

Let $R(D ; \sigma, \rho)$ denote the rate distortion function of sending a bivariate zero-mean Gaussian source with symmetric covariance matrix $\left[\begin{array}{cc}\sigma^{2} & \rho \sigma^{2} \\ \rho \sigma^{2} & \sigma^{2}\end{array}\right]$

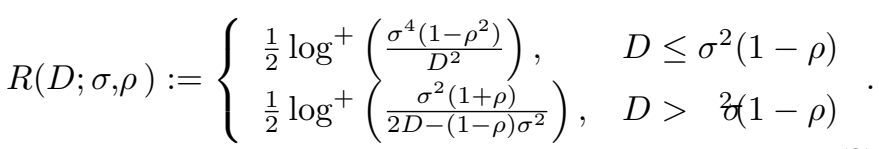

Below we give a necessary condition for $D^{s}$ to be achievable in Fig. 2(a).

Theorem 1: If $D^{s}$ is achievable, then

$$
R\left(D^{s} ; \sigma_{v}, \rho_{v}\right) \leq \frac{1}{2} \log \left(1+2\left(1+\rho_{s}\right) \eta_{\mathrm{c}}\right),
$$

where $\sigma_{v}=\sigma_{s}$ and $\rho_{v}=\rho_{s}+2 \alpha \beta\left(1-\rho_{s}^{2}\right)$.

Proof: Consider an enhanced system in Fig. 2(b) where the two encoders merge into a joint encoder. If $D^{s}$ is achievable in the original system, so is it in the enhanced system. Since $\alpha \neq \beta$, we can further see that it is equivalent to the system in Fig. 2(c), where the encoder takes $v_{1}$ and $v_{2}$ as inputs.

Apply the converse argument in [4] (where Witsenhausen's maximal correlation property [9] is used) to show that for the original system, the maximum power of the aggregate transmission of the two transmitters is $P_{1}+P_{2}+2 \rho_{s} \sqrt{P_{1} P_{2}}=$ $2 P\left(1+\rho_{s}\right)$. Hence for the enhanced system in Fig. 2(b) and (c), the power constraint at the transmitter is $2 P\left(1+\rho_{s}\right)$.

In the enhanced system in Fig. 2(c), the optimal distortion is known to satisfy (3) due to the optimality of source-channel separation in point-to-point settings and the calculations done in [4].

The following corollary gives the high-SNR asymptotic behavior of the lower bound on the achievable distortion derived from the previous theorem. Its proof is straightforward so we omit it.

Corollary 1: Denote the distortion lower bound in Theorem 1 by $\underline{D}^{s}$. Then

$$
\lim _{\eta_{\mathrm{c}} \rightarrow \infty} \sqrt{\eta_{\mathrm{c}}} \underline{D}^{s}=\sigma_{s}^{2} \sqrt{\frac{1-\rho_{s}}{2}}\left|\alpha^{2}-\beta^{2}\right| .
$$

In the next subsection, we shall see that this high-SNR asymptotic lower bound is, in fact, achievable.

\section{B. Inner Bounds}

We first present an inner bound based on uncoded transmission.

Theorem 2 (Uncoded Scheme Inner Bound): A sufficient condition for $D^{s}$ to be achievable is

$$
D^{s} \geq \sigma_{s}^{2}\left\{\frac{1+\eta_{\mathrm{c}}(\alpha-\beta)^{2}\left(1-\rho_{s}^{2}\right)}{1+2 \eta_{\mathrm{c}}\left(1+\rho_{s}\right)}\right\} .
$$

Proof: Each encoder simply scales its observation to match the power constraint and sends it over the channel. The resulting end-to-end distortion can be calculated easily because all the random variables are jointly Gaussian.

Remark 1: The optimality of the uncoded lower bound is obtained whenever the above distortion upper bound

$$
\begin{aligned}
& \sigma_{s}^{2}\left\{\frac{1+\eta_{\mathrm{c}}(\alpha-\beta)^{2}\left(1-\rho_{s}^{2}\right)}{1+2 \eta_{\mathrm{c}}\left(1+\rho_{s}\right)}\right\}>\sigma_{v}^{2}\left(1-\rho_{v}\right) \\
\Longleftrightarrow & \eta_{\mathrm{c}}<\frac{\rho_{s}+2 \alpha \beta\left(1-\rho_{s}^{2}\right)}{(\alpha-\beta)^{2}\left(1-\rho_{s}^{2}\right)}
\end{aligned}
$$

since $\sigma_{v}^{2}\left(1-\rho_{v}\right)=\sigma_{s}^{2}\left(1-\rho_{s}^{2}\right)(\alpha-\beta)^{2}$. Hence, we characterize the channel SNR threshold below which uncoded scheme is optimal. Note that the threshold given in (6) depends on $\rho_{s}, \alpha$, and $\beta$. Note that when $\alpha \beta=0$, we reproduce the result in [4]. When $\alpha=\beta$ we reproduce the result in [8], where uncoded scheme is shown to be optimal.

Next we want to explore the high-SNR asymptotic performance. In [4], it is shown that a vector-quantization scheme achieves the high-SNR asymptotic performance in the direct Gaussian joint source channel coding problem over Gaussian MAC. The following theorem extends this result to the remote setting (equivalently, direct setting with linear functions as 


$$
\begin{aligned}
2 R_{s} & \leq \frac{1}{2} \log \left(\frac{K_{\bar{z}}+2\left(\gamma_{\mathrm{d}}+\gamma_{\mathrm{a}} \frac{1+\rho_{s}}{1+\rho_{s}\left(1-2^{-2 R_{s}}\right)}\right)^{2} \sigma_{s}^{2}\left(1-2^{-2 R_{s}}\right)\left(1+\rho_{s}\left(1-2^{-2 R_{s}}\right)\right)}{K_{\bar{z}}\left(1-\rho_{s}^{2}\left(1-2^{-2 R_{s}}\right)^{2}\right)}\right) \\
& 2 R_{s}^{*}=\frac{1}{2} \log \left(\frac{2 \eta_{\mathrm{c}}\left(1+\rho_{s}\left(1-2^{-2 R_{s}^{*}}\right)\right)}{\left(1-\rho_{s}^{2}\left(1-2^{-2 R_{s}^{*}}\right)^{2}\right)+\kappa \eta_{\mathrm{c}}^{\lambda}\left(1+\rho_{s}\right)\left(1-\rho_{s}\left(1-2^{-2 R_{s}^{*}}\right)\right) 2^{-2 R_{s}^{*}}}\right) \\
\Longleftrightarrow & 2^{-2 R_{s}^{*}}=\sqrt{\frac{1-\rho_{s}\left(1-2^{-2 R_{s}^{*}}\right)}{2}\left[1+\frac{\left(1+\rho_{s}\right)\left(\kappa \eta_{\mathrm{c}}^{\lambda} 2^{-2 R_{s}^{*}}\right)}{1+\rho_{s}\left(1-2^{-2 R_{s}^{*}}\right)}\right]} \frac{1}{\sqrt{\eta_{\mathrm{c}}}} .
\end{aligned}
$$

targets of reconstruction), and shows that the coding system has to be re-designed for the remote setting.

Before stating the theorem, we start with the description of the scheme. The scheme we use is the superposition scheme proposed in Section IV-E of [4]. Such superposition scheme also appears in [10] for sending a Gaussian source over a Gaussian point-to-point channel and in [3] for sending jointly Gaussian sources over Gaussian broadcast channel, and it is recently extended in [11] to a more general setting. Each encoder, say, $i$, first vector-quantizes its observation $\mathbf{s}_{i}$ using a rate- $R_{i}$ code and obtains $\mathbf{u}_{i}$, for $i=1,2$. Then each transmitter, say Tx $i$, transmits a linear combination of its observation $\mathbf{s}_{i}$ and the VQ output $\mathbf{u}_{i}$, so that the overall transmission from Tx $i$ is

$$
\mathbf{x}_{i}=\gamma_{\mathrm{a}} \mathbf{s}_{i}+\gamma_{\mathrm{d}} \mathbf{u}_{i}, \quad i=1,2,
$$

where the subscript "a" and "d" denote analog and digital respectively. The vector quantization code has rate $R_{s}$. Based on the analysis of [4], the power constraint at each transmitter implies

$$
\begin{aligned}
& \gamma_{\mathrm{a}}^{2} \sigma_{s}^{2}+\gamma_{\mathrm{d}}^{2} \sigma_{s}^{2}\left(1-2^{-2 R_{s}}\right)+2 \gamma_{\mathrm{a}} \gamma_{\mathrm{d}} \sigma_{s}^{2}\left(1-2^{-2 R_{s}}\right) \leq P \\
\Longleftrightarrow & 2^{-2 R_{s}} \gamma_{\mathrm{a}}^{2}+\left(1-2^{-2 R_{s}}\right)\left(\gamma_{\mathrm{a}}+\gamma_{\mathrm{d}}\right)^{2} \leq \frac{P}{\sigma_{s}^{2}} .
\end{aligned}
$$

Note that $\gamma_{\mathrm{a}}$ and $\gamma_{\mathrm{d}}$ determines the power split between the "analog" (uncoded transmission) part and the "digital" (vectorquantized) part.

Following the same line of analysis as in [4], we obtain the following theorem regarding the achievable distortion of the superposition scheme.

Theorem 3: Let $D_{\text {super }}^{s}$ denote the achievable distortion by the superposition scheme. For any $\gamma_{\mathrm{a}}, \gamma_{\mathrm{d}}, R_{s}$ satisfying (7) and the constraint (4) on the top of this page, where $K_{\bar{z}}:=N_{o}+2 \gamma_{\mathrm{a}}^{2} \sigma_{s}^{2}\left[\frac{1+\rho_{s}}{1+\rho_{s}\left(1-2^{-2 R_{s}}\right)}\right] 2^{-2 R_{s}}$, we have the following achievable distortion $D_{\text {super }}^{s}=D_{\mathrm{VQ}}^{s}-\mathcal{I}^{s}$, where $D_{\mathrm{VQ}}^{s}=\sigma_{s}^{2}\left\{\frac{2^{-2 R_{s}}+\left(1-\rho_{s}^{2}\right)\left(1-2^{-2 R_{s}}\right)\left(\alpha^{2}+\beta^{2}\right)}{1-\rho_{s}^{2}\left(1-2^{-2 R_{s}}\right)^{2}}\right\} 2^{-2 R_{s}}$ and $\mathcal{I}^{s}=\sigma_{s}^{2}\left\{\frac{\gamma_{\mathrm{a}}^{2} \sigma_{s}^{2}(\alpha+\beta)^{2}\left(\frac{1+\rho_{s}}{1+\rho_{s}\left(1--^{-2 R s}\right)}\right)^{2} 2^{-4 R_{s}}}{N_{o}+2 \gamma_{\mathrm{a}}^{2} \sigma_{s}^{2}\left(\frac{1+\rho_{s}}{1+\rho_{s}\left(1-2^{-2 R_{s}}\right)}\right) 2^{-2 R_{s}}}\right\}$.

Proof: The proof follows from the analysis of decoding error probability in [4], along with a straightforward calculation of MMSE estimation error.
Note that the above power constraint (7) allows $\gamma_{\mathrm{a}} \gamma_{\mathrm{d}}<0$, but we shall constrain to $\left(\gamma_{\mathrm{a}}, \gamma_{\mathrm{d}}\right)$ satisfying (7) with equality and

$$
0 \leq \gamma_{\mathrm{a}} \leq \sqrt{\frac{P}{\sigma_{s}^{2}}}, 0 \leq \gamma_{\mathrm{d}} \leq \sqrt{\frac{P}{\sigma_{s}^{2}\left(1-2^{-2 R_{s}}\right)}}
$$

in the rest of this paper, and show that a proper choice of the power split can achieve the high-SNR asymptotic distortion lower bound given in Corollary 1.

To proceed, we first simplify the rate constraint (4) and pick a manageable $R_{s}^{*}$ satisfying (4). It is not hard to evaluate that the right-hand-side of (4) is equal to $\frac{1}{2} \log \left(\frac{N_{o}+2\left(1+\rho_{s}\right) P-2 \gamma_{\mathrm{d}}^{2} \sigma_{s}^{2}\left(1-2^{-2 R_{s}}\right) \rho_{s} 2^{-2 R_{s}}}{N_{o}\left(1-\rho_{s}^{2}\left(1-2^{\left.-2 R_{s}\right)^{2}}\right)+2 \gamma_{\mathrm{a}}^{2} \sigma_{s}^{2}\left(1+\rho_{s}\right)\left(1-\rho_{s}\left(1-2^{-2 R_{s}}\right)\right) 2^{-2 R_{s}}\right.}\right)$. Next, we lower bound this term by plugging $\gamma_{\mathrm{d}}=\sqrt{\frac{P}{\sigma_{s}^{2}\left(1-2^{\left.-2 R_{s}\right)}\right.}}$ into the numerator:

$$
\begin{aligned}
& N_{o}+2\left(1+\rho_{s}\right) P-2 \gamma_{\mathrm{d}}^{2} \sigma_{s}^{2}\left(1-2^{-2 R_{s}}\right) \rho_{s} 2^{-2 R_{s}} \\
& \geq N_{o}+2 P\left(1+\rho_{s}\left(1-2^{-2 R_{s}}\right)\right) \\
& \geq 2 P\left(1+\rho_{s}\left(1-2^{-2 R_{s}}\right)\right) .
\end{aligned}
$$

We shall choose $\gamma_{\mathrm{a}}$ such that $2 \gamma_{\mathrm{a}}^{2} \sigma_{s}^{2} / N_{o}=\kappa \eta_{\mathrm{c}}^{\lambda}$, where $0 \leq \kappa \leq 2 \eta_{\mathrm{c}}^{1-\lambda}$ and $0 \leq \lambda \leq 1$. In other words, we set the SNR of the uncoded part scale as $\Theta\left(\eta_{c}^{\lambda}\right)$ and satisfy (8). With such choice and the lower bounding procedure above, we pick $R_{s}^{*}$ such that it satisfies (5) on the top of this page.

With different choices of $\lambda$, the scaling of $2^{-2 R_{s}^{*}}$ with respect to $\eta_{\mathrm{c}}$ will be different. The following lemma summarizes the behavior.

Lemma 2: $2^{-2 R_{s}^{*}}=\Theta\left(\eta_{\mathrm{c}}^{\max \{-1 / 2, \lambda-1\}}\right)$

Proof: It is straightforward to see that rate $R_{s}^{*} \rightarrow$ $\infty$ as $\eta_{\mathrm{c}} \rightarrow \infty$. Hence, $2^{-2 R_{s}^{*}} \rightarrow 0$ as $\eta_{\mathrm{c}} \rightarrow \infty$. Therefore, as $\eta_{\mathrm{c}} \rightarrow \infty$, the right-hand-side (RHS) of (5) approaches $\sqrt{\frac{\left(1-\rho_{s}\right)\left(1+\kappa \eta_{\mathrm{c}}^{\lambda} 2^{\left.-2 R_{s}^{*}\right)}\right.}{2 \eta_{\mathrm{c}}}} \sim \sqrt{\frac{\left(1+\kappa \eta_{\mathrm{c}}^{\lambda} 2^{\left.-2 R_{s}^{*}\right)}\right.}{\eta_{\mathrm{c}}}} \sim$ $\sqrt{\frac{\max \left(1, \kappa \eta_{\mathrm{c}}^{\lambda} 2^{-2 R_{s}^{*}}\right)}{\eta_{\mathrm{c}}}}$. Hence $2^{-2 R_{s}^{*}}=\Theta\left(\eta_{\mathrm{c}}^{\max \{-1 / 2, \lambda-1\}}\right)$.

We shall make use of this lemma to show that, only the choice $\lambda=1 / 2$ can attain the distortion lower bound given in Corollary 1. Moreover, there is a unique choice of $\kappa$ that can attain it. This result is summarized in the following theorem. 
Theorem 4: The following choice of $\lambda$ and $\kappa$ is the only one that can attain the distortion lower bound in Corollary 1 :

$$
\lambda=\frac{1}{2}, \kappa=\sqrt{\frac{2}{1-\rho_{s}}}\left(\frac{4 \alpha \beta}{\left|\alpha^{2}-\beta^{2}\right|}\right)
$$

Proof: If $0 \leq \lambda<1 / 2$, by Lemma 2 we have $2^{-2 R_{s}^{*}}=$ $\Theta\left(\eta_{\mathrm{c}}^{-1 / 2}\right)$ and hence $\kappa \eta_{\mathrm{c}}^{\lambda} 2^{-2 R_{s}^{*}} \rightarrow 0$ as $\eta_{\mathrm{c}} \rightarrow \infty$. Therefore,

$$
\begin{aligned}
D_{\mathrm{VQ}}^{s} & =\sigma_{s}^{2}\left\{\frac{2^{-2 R_{s}^{*}}+\left(1-\rho_{s}^{2}\right)\left(1-2^{-2 R_{s}^{*}}\right)\left(\alpha^{2}+\beta^{2}\right)}{1-\rho_{s}^{2}\left(1-2^{-2 R_{s}^{*}}\right)^{2}}\right\} 2^{-2 R_{s}^{*}} \\
& =\Theta\left(\eta_{\mathrm{c}}^{-1 / 2}\right), \\
\mathcal{I}^{s} & =\sigma_{s}^{2}\left\{\frac{\frac{N_{o}}{2} \kappa \eta_{\mathrm{c}}^{\lambda}(\alpha+\beta)^{2}\left(\frac{1+\rho_{s}}{1+\rho_{s}\left(1-2^{-2 R_{s}^{*}}\right)}\right)^{2} 2^{-4 R_{s}^{*}}}{N_{o}+N_{o} \kappa \eta_{c}^{\lambda}\left(\frac{1+\rho_{s}}{1+\rho_{s}\left(1-2^{-2 R_{s}^{*}}\right)}\right) 2^{-2 R_{s}^{*}}}\right\} \\
& =\Theta\left(\eta_{\mathrm{c}}^{\lambda-1}\right) .
\end{aligned}
$$

Hence $D_{\text {super }}^{s}=\Theta\left(\eta_{\mathrm{c}}^{-1 / 2}\right)$. Then, $\lim _{\eta_{\mathrm{c}} \rightarrow \infty} \sqrt{\eta_{\mathrm{c}}} D_{\text {super }}^{s}=$ $\lim _{\eta_{\mathrm{c}} \rightarrow \infty} \sqrt{\eta_{\mathrm{c}}} D_{\mathrm{VQ}}^{s}$ and

$$
\begin{aligned}
& \lim _{\eta_{\mathrm{c}} \rightarrow \infty} \sqrt{\eta_{\mathrm{c}}} D_{\mathrm{VQ}}^{s}=\sigma_{s}^{2}\left(\alpha^{2}+\beta^{2}\right) \lim _{\eta_{\mathrm{c}} \rightarrow \infty} \sqrt{\eta_{\mathrm{c}}} 2^{-2 R_{s}^{*}} \\
& =\sigma_{s}^{2}\left(\alpha^{2}+\beta^{2}\right) \sqrt{\frac{1-\rho_{s}}{2}}>\sigma_{s}^{2} \sqrt{\frac{1-\rho_{s}}{2}}\left|\alpha^{2}-\beta^{2}\right| .
\end{aligned}
$$

We conclude that it cannot achieve the distortion lower bound.

If $1 / 2<\lambda \leq 1$, by Lemma 2 we have $2^{-2 R_{s}^{*}}=\Theta\left(\eta_{\mathrm{c}}^{\lambda-1}\right)$. Hence $D_{\mathrm{VQ}}^{s}=\Theta\left(\eta_{\mathrm{c}}^{\lambda-1}\right)$ and $\mathcal{I}^{s}=\Theta\left(\eta_{\mathrm{c}}^{\lambda-1}\right)$. Therefore, $D_{\text {super }}^{s}=\Theta\left(\eta_{\mathrm{c}}^{\lambda-1}\right)$ and cannot achieve even the same scaling as the distortion lower bound in Corollary 1.

From the above discussion, we conclude that $\lambda$ has to be $1 / 2$. With $\lambda=1 / 2$, note that since $2^{-2 R_{s}^{*}}=\Theta\left(\eta_{\mathrm{c}}^{-1 / 2}\right)$ and $\sqrt{\eta_{\mathrm{c}}} 2^{-2 R_{s}^{*}}=\sqrt{\frac{1-\rho_{s}\left(1-2^{-2 R_{s}^{*}}\right)}{2}\left[1+\frac{\left(1+\rho_{s}\right)\left(\kappa \eta_{c}^{\lambda} 2^{-2 R_{s}^{*}}\right)}{1+\rho_{s}\left(1-2^{-2 R_{s}^{*}}\right)}\right]}$, we see that $\lim _{\eta_{\mathrm{c}} \rightarrow \infty} \sqrt{\eta_{\mathrm{c}}} 2^{-2 R_{s}^{*}}=X^{*}$ where $X^{*}$ is the positive root of the quadratic equation $X^{2}=\frac{1-\rho_{s}}{2}(1+\kappa X)$. It is easy to solve it and get $\lim _{\eta_{\mathrm{c}} \rightarrow \infty} \sqrt{\eta_{\mathrm{c}}} 2^{-2 R_{s}^{*}}=X^{*}=\frac{1-\rho_{s}}{4} \kappa+$ $\sqrt{\frac{1-\rho_{s}}{2}+\frac{\left(1-\rho_{s}\right)^{2}}{16} \kappa^{2}}$.

The corresponding distortion asymptotic is

$$
\begin{aligned}
& \left.\begin{array}{l}
\lim _{\eta_{\mathrm{c}} \rightarrow \infty} \sqrt{\eta_{\mathrm{c}}} D_{\mathrm{VQ}}^{s}=\sigma_{s}^{2} X^{*}\left(\alpha^{2}+\beta^{2}\right) \\
\lim _{\eta_{\mathrm{c}} \rightarrow \infty} \sqrt{\eta_{\mathrm{c}}} \mathcal{I}^{s}=\sigma_{s}^{2} X^{*}\left(\frac{\frac{1}{2}(\alpha+\beta)^{2} \kappa X^{*}}{1+\kappa X^{*}}\right)
\end{array}\right\} \Rightarrow \\
& \lim _{\eta_{\mathrm{c}} \rightarrow \infty} \sqrt{\eta_{\mathrm{c}}} D_{\text {super }}^{s}=\sigma_{s}^{2} X^{*}\left(\alpha^{2}+\beta^{2}-\frac{\frac{1}{2}(\alpha+\beta)^{2} \kappa X^{*}}{1+\kappa X^{*}}\right) .
\end{aligned}
$$

To obtain the distortion lower bound, we need to find $\kappa$ satisfying $\sqrt{\frac{1-\rho_{s}}{2}}\left|\alpha^{2}-\beta^{2}\right|=X^{*}\left(\alpha^{2}+\beta^{2}-\frac{\frac{1}{2}(\alpha+\beta)^{2} \kappa X^{*}}{1+\kappa X^{*}}\right)$. Plug in $\left(X^{*}\right)^{2}=\frac{1-\rho_{s}}{2}\left(1+\kappa X^{*}\right)$ and after some manipulations, we arrive at $\left(X^{*}-\frac{\alpha+\beta}{|\alpha-\beta|} \sqrt{\frac{1-\rho_{s}}{2}}\right)^{2}=0$, which implies that we should take $X^{*}=\frac{\alpha+\beta}{|\alpha-\beta|} \sqrt{\frac{1-\rho_{s}}{2}}$ to attain the distortion lower bound. Equivalently, the only value of $\kappa$ that we should take is $\kappa^{*}=\sqrt{\frac{2}{1-\rho_{s}}}\left(\frac{4 \alpha \beta}{\left|\alpha^{2}-\beta^{2}\right|}\right)$. Proof complete.
We conclude this section by a remark on the optimal superposition scheme in high-SNR regime. Note that if $\alpha \beta=0$, we are back to the problem studied in [4], which is reconstructing the observations at the decoder. In this case, we see that the optimal $\kappa^{*}=0$, meaning that the simply the vectorquantization scheme is sufficient to achieve the optimal highSNR asymptotic performance.

\section{CONCluding Remarks}

Estimation-coding separation provides an elegant solution to remote joint source-channel coding in the point-to-point setting. When more encoders in the system take noisy measurements of correlated sources, such separation is no longer optimal. In this paper we investigated the problem of communicating two remote jointly Gaussian sources over a two-user Gaussian MAC. We showed that in the Gaussian setting, the problem is equivalent to reconstructing two linear combinations of the observation (noisy measurements of the remote sources). We characterized the optimal achievable distortion up to a certain SNR threshold, where uncoded transmission is optimal. We also characterized the high-SNR asymptotic of the optimal distortion, where a superposition of an uncoded scheme and a vector-quantizor scheme is required. Moreover, we showed that in contrast to the direct problem studied in [4], a significant amount of power should be allocated to uncoded transmission: its effective SNR should scale as the square-root of the overall SNR as the SNR tends to infinity.

\section{REFERENCES}

[1] C. E. Shannon, "Coding theorems for a discrete source with a fidelity criterion," IRE International Convention Record, vol. 7, pp. 142-163, 1959.

[2] S. Bross, A. Lapidoth, and S. Tinguely, "Broadcasting correlated Gaussians," IEEE Transations on Information Theory, vol. 56, pp. 3057-3068, July 2010.

[3] C. Tian, S. Diggavi, and S. Shamai, "The achievable distortion region of bivariate Gaussian source on Gaussian broadcast channel," Submitted to IEEE Transations on Information Theory, June 2010, http://arxiv.org/ abs/1006.0644.

[4] A. Lapidoth and S. Tinguely, "Sending a bivariate Gaussian over a Gaussian MAC," IEEE Transations on Information Theory, vol. 56, pp. 2714-2752, June 2010.

[5] R. L. Dobrushin and B. S. Tsybakov, "Information transmission with additional noise," IRE Transactions on Information Theory, vol. IT-18, pp. S293-S304, 1962.

[6] J. K. Wolf and J. Ziv, "Transmission of noisy information to a noisy receiver with minimum distortion," IEEE Transations on Information Theory, vol. IT-16, pp. 406-411, July 1970.

[7] A. B. Wagner, S. Tavildar, and P. Viswanath, "Rate region of the quadratic Gaussian two-encoder source-coding problem," IEEE Transations on Information Theory, vol. 54, pp. 1938-1961, May 2008.

[8] M. Gastpar, "Uncoded transmission is exactly optimal for a simple Gaussian "sensor" network," IEEE Transations on Information Theory, vol. 54, pp. 5247-5251, November 2008.

[9] H. S. Witsenhausen, "On sequences of pairs of dependent random variables," SIAM Journal on Applied Mathematics, vol. 28, pp. 100113, January 1975.

[10] S. Bross, A. Lapidoth, and S. Tinguely, "Superimpoed coded and uncoded transmission of a Gaussian source over the Gaussian channel," Proceedings of IEEE International Symposium on Information Theory, July 2006.

[11] S. H. Lim, P. Minero, and Y.-H. Kim, "Lossy communication of correlated sources over multiple access channels," Proceedings of Allerton Conference on Communication, Control, and Computing, September 2010. 